\title{
Developing a spinal cord injury research strategy
}

\author{
JJ Wyndaele \\ Antwerp, Belgium \\ E-mail: wyndaelejj@skynet.be
}

Most will agree that progress in knowledge and comprehensive management of SCI depend largely on research. But which research is considered most interesting, most valuable and most readable, and how should it be structured? All of these factors are important to evaluate and consider. This issue contains 6 manuscripts dealing with this topic.

Research: In part I of the 3 bundled manuscripts, Bragge et al. made a worldwide literature search to gain an overview of the volume, nature and findings of studies regarding priorities for SCI research. Despite the difference in research paradigms, they found convergence in the areas of body impairments and relationships. The vast majority of literature within the reviews focused on the SCI patient perspective. In part II, Bragge et al. present a research strategy 'roadmap' developed to define the major steps and key planning questions to consider. Evidence from published SCI research strategy initiatives was synthesised with information from 4 one-on-one semistructured interviews with key SCI research stakeholders to create a research strategy framework, articulating six key themes and associated activities for consideration. These resources, combined with a review of SCI prioritisation literature, were used to generate a list of draft principles for discussion in a structured stakeholder dialogue meeting. In part III, entitled 'outcomes', Middleton et al. propose a regional SCI research strategy, whose objectives can be summarised under four themes:

(1) Collaborative networks and strategic partnerships to increase efficiency, reduce duplication, build capacity and optimise research funding

(2) Research priority setting and coordination to manage competing studies

(3) Mechanisms for greater consumer engagement in research

(4) Resources and infrastructure to further develop SCI data registries, evaluate research translation and assess alignment of research strategy with stakeholder interests

Three more manuscripts give practical details of conducting and planning research: Krebs et al. evaluated study participation rate of patients with acute SCI early during rehabilitation. The approximately $50 \%$ study participation rate needs to be considered when planning clinical trials. Individuals older than 60 years participated much less. Gollan et al. describe the different steps of development, determination of reliability and validity of the Queensland Evaluation of Wheelchair Skills. Garcia-Masso et al. developed and tested classification algorithms based on machine learning, using accelerometers to identify the activity type performed by manual wheelchair users with SCI.

Outcome: Liu et al. found in a retrospective study that early MRI added little in the determination of stability of the cervical spine in the presence of a negative CT (SCIWORA). The study suggests that the neurological outcome did not correlate with early MRI findings. Lechler and Boese state in a comment that these findings contradict the majority of the previously published literature in adults and children. They are convinced that the inclusion of precisely classified early MRI findings as well as the presentation of the AIS at admission, discharge and final follow up on individual level should be the minimum standard for future reports on SCIWORA. Kepler et al. found chronic hypertension to be an independent risk factor for poor early neurologic recovery in patients with acute SCI, independent of age and other co-morbidities.

Locomotor training: Arazpour et al. evaluate the influence of walking with an isocentric reciprocating gait orthosis on different parameters of walking in a small series. Nooijen et al. determined the prevalence and severity of fatigue in persons with subacute spinal cord injury.

Pressure ulcers: Kovindha et al. found pressure ulcers to still be prevalent among Thai wheelchair users with chronic SCI. Anxiety/depression is associated with current ulcers.

Please also check out our new spin-off journal: Spinal Cord: Series and Cases (www.nature.com/scsandc), the best forum for cases and small series.

Spinal Cord (2015) 53, 713; doi:10.1038/sc.2015.163 